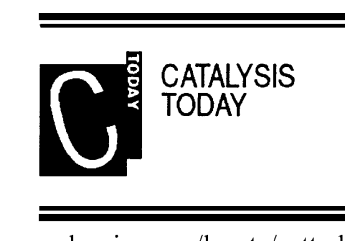

Catalysis Today 79-80 (2003) 1

www.elsevier.com/locate/cattod

\title{
Editorial
}

\section{Preface: catalysis in multiphase reactors}

This volume contains the material presented at the 4th International Symposium on Catalysis in Multiphase Reactors (CAMURE-4, 22-25 September 2002) in Lausanne, Switzerland. Divers chemical processes involve multiphase catalytic reactions useful in the manufacture of intermediates and consumer end-products like pharmaceuticals, commodity and specialty chemicals, fuels, hydrocarbons, polymers, herbicides and pesticides. Recently multiphase catalytic reactors have been used in pollution abatement and for environmental protection. Various emerging chemistries demand multiphase reactor technology. For rapid development and commercialization of new technologies an appropriate choice of multiphase reactor together with understanding of scale-up principles is necessary.

The new paradigm in modern multiphase chemical reaction engineering is based on the simultaneous development of the catalyst and the reactor. The catalyst design is closely integrated with the reactor design taking into consideration the energy requirements of the process and the reaction mechanism with respect to selectivity/yield of the target product. This new approach led to the application of structured catalysts and reactors for multiphase processes.

In order to make an appropriate reactor selection and also guide the catalyst development with desirable formulation, suitable size, shape and morphology, one needs to consider fluid dynamics, flow mixing patterns, transport phenomena, contact phases pattern, wetting and particle scale phenomena. The success of the whole process development and its scale-up depends strongly on the reactor modeling. Recent rapid advances in available software for computational fluid dynamics (CFD) allow simulating flow patterns in large reactors in cold model. Detailed models based on the chemistry and transport phe- nomena in multiphase reactors are still to be worked out.

All of these factors make multiphase catalytic reaction engineering an interdisciplinary field. The broad area of subjects in the agenda led to a high attendance of the CAMURE-4 symposium. About 150 delegates from 27 countries participated in this symposium including scientists and engineers from industry, academia and research institutions. The participants reported the most significant achievements and ongoing efforts in the particular field. The manuscripts in this volume were reviewed by experts and followed by the author's revisions to incorporate their suggestions. We hope that the material presented will be of value for the scientific and technical community and will stimulate further research, design and application.

Finally, we thank the members of Scientific Committee and also reviewers who carried out the refereeing process. We also express our gratitude to the authors for their contributions. The financial support of the Swiss National Science Foundation, the Swiss Society for Chemical Engineering, the Swiss Federal Institute of Technology, Lausannne, as well as from different industrial organizations (CMD International, Nestlé, Rhodia, Roche Vitamins, Sulzer Chemtech) is appreciated.

Lioubov Kiwi-Minsker*, Albert Renken Laboratory of Chemical Reaction Engineering (LGRC), Institute of Process Science Swiss Federal Institute of Technology (EPFL) CH-1015 Lausanne, Switzerland

${ }^{*}$ Corresponding author. Tel.: +41-21-693-3182 fax: $+41-21-693-3190$

E-mail addresses: lioubov.kiwi-minsker@epfl.ch (L. Kiwi-Minsker) albert.renken@epfl.ch (A. Renken) 